\title{
Global Doppler frequency shift detection with near-resonant interferometry
}

\section{Journal Article}

Author(s):

Landolt, Andrin; Rösgen, Thomas

Publication date:

2009-10

Permanent link:

https://doi.org/10.3929/ethz-b-000058929

Rights / license:

In Copyright - Non-Commercial Use Permitted

Originally published in:

Experiments in Fluids 47(4-5), https://doi.org/10.1007/s00348-009-0688-4 


\title{
Global Doppler frequency shift detection with near-resonant interferometry
}

\author{
Andrin Landolt · Thomas Roesgen
}

Received: 1 October 2008/Revised: 12 May 2009/Accepted: 14 May 2009/Published online: 2 June 2009

(C) Springer-Verlag 2009

\begin{abstract}
The recent development in measuring 2D Doppler shift distributions for flow velocimetry using the dispersive properties of atomic line filters is presented. On the basis of velocity field measurements on a subsonic jet flow and a tip vortex flow in a medium-sized wind tunnel, the technique was assessed. Atomic line filters near a resonant transition combine imaging capabilities with a sharp frequency cutoff and an associated region of strong anomalous dispersion. While conventional Doppler global velocimetry relies on the absorption of the filter to convert frequency shifts to intensity variations, near-resonant interferometry uses its dispersion to detect frequency shifts as phase changes in an interference pattern. In the present setup, an iodine vapor cell in an imaging Michelson interferometer is used. With the illuminating laser frequency tuned near a resonant transition, the cell's dispersion converts the frequency content of the field of view into a distortion of the carrier-fringe pattern recorded at the image plane of the interferometer. The phase distribution in the fringe images is reconstructed by filtering the individual images with a $2 \mathrm{D}$ Gabor filter pair tuned to the spatial frequencies of the basic carrier-fringe pattern. The post-processing is concluded with subsequent phase-unwrapping and subtraction of the carrier reference fringe phase. The method and the setup were demonstrated and calibrated experimentally on a rotating disc. The capability of the technique to operate in a real experimental environment was validated in a free subsonic jet and a tip vortex flow behind a wing section in a mediumsized wind tunnel facility. The measurements were found to
\end{abstract}

\footnotetext{
A. Landolt $(\bowtie) \cdot T$. Roesgen

Institute of Fluid Dynamics, ETH, Zurich, Switzerland

e-mail: landolt@ifd.mavt.ethz.ch

T. Roesgen

e-mail: roesgen@ifd.mavt.ethz.ch
}

be in generally good agreement with the theoretically predicted system characteristics and the reference measurements. As with other Doppler global techniques, the stability of the pulsed laser system and the secondary scattering in the test volume were identified as the main error sources.
Abbreviations
ALF Atomic line filter
AOA Angle of attack
DGV Doppler global velocimetry
DPV Doppler picture velocimetry
FWHM Full width at half maximum
ICCD Intensified charge-coupled device
LDV Laser Doppler velocimetry
PDV Planar Doppler velocimetry
PIV Particle image velocimetry
RMS Root mean square

\section{Introduction}

Doppler global imaging techniques measure the shift in the frequency of light scattered by clouds of moving tracer particles to determine the flow velocity field. The measured component thereby depends on the direction of illumination $l$ and the viewing direction of the observer $o$ (Fig. 1) as described by the Doppler relation (1) for velocities $V$ that are small compared to the speed of light $c$ :

$\Delta v=v_{0} \frac{\underline{o}-\underline{l}}{c} \underline{V}$,

where $\Delta v$ is the measured frequency shift and $v_{0}$ the emission frequency of the light the source. Compared to 


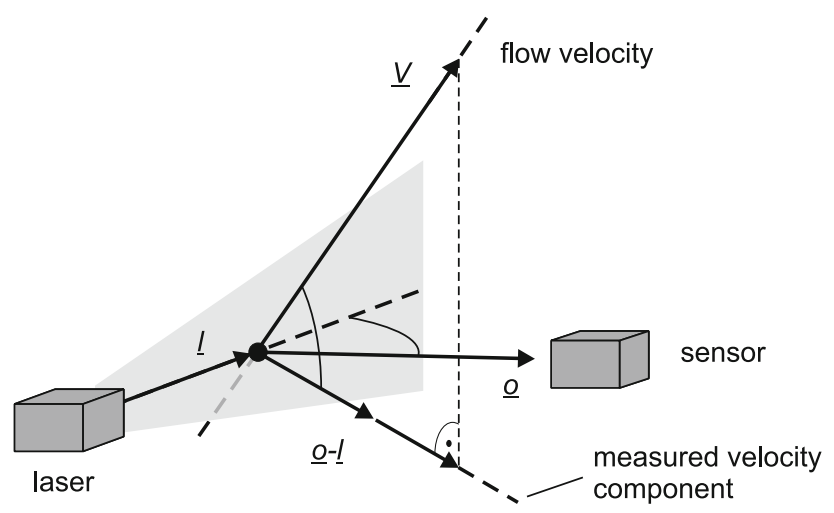

Fig. 1 Principle of Doppler global techniques

scanning the region of interest using a Laser Doppler Velocimeter (LDV), the acquisition of the data is much faster. Without the need to resolve individual tracer particles, a larger field of view can be recorded than with particle image velocimetry (PIV). Unlike PIV, the method is sensitive to the out-of-plane component of the illuminated flow field. Doppler imaging techniques are hence well suited for large-scale applications or where optical access prevents the use of PIV.

In Doppler global velocimetry (DGV, also planar Doppler velocimetry, PDV) the absorption property of an atomic line filter (ALF) is used to transform the Doppler shift into intensity distribution (Komine et al. 1991; Meyers 1995). The variations in illumination intensity and scattering signal are eliminated by normalizing the recorded filtered measurement image with a reference image. Thus, DGV is typically a two-camera technique with stringent requirements concerning image alignment and camera calibration. High dynamic range and linearity of the image sensors are required. Current systems are capable of reaching an absolute measurement error of about $1 \mathrm{~ms}^{-1}$ (Meyers et al. 2001; Willert et al. 2007). Consequently, DGV is still regarded as more suited for high velocity applications.

Different single-camera adaptations of global Doppler velocimetry have been presented recently. A dual frequency technique was developed by Ford et al. (2001). The flow is illuminated sequentially by two beams of the same laser but at different frequencies. The signal image is recorded with the beam frequency on an absorption line of an ALF, while the beam that illuminates the reference image is shifted to a region of zero absorption. The performance achieved on a rotating disc and later on a free jet was comparable to conventional DGV. An alternative single sensor technique based on frequency modulation (FM-DGV) was investigated by Fischer et al. (2007). The data recorded by avalanche photodiode array on a rotating disc and a jet flow shows very high accuracy at the cost (so far) of spatial resolution. Meier and Roesgen (2009) introduced heterodyne Doppler global velocimetry (HDGV) where the light scattered by the flow tracers is heterodyned with a reference beam from the same light source. The signal is detected using a single smart pixel imaging array that performs a lock-in detection on each pixel. Limited by the maximum demodulation frequency of the sensor, the application is so far restricted to small velocities.

An interferometric approach was presented by Seiler and Oertel (1983) with Doppler picture velocimetry (DPV). The technique relies on an imaging Michelson interferometer with large path length difference. Because of the difference in the optical path length, frequency shifts are transformed to local distortions in the carrierfringe pattern at the image plane of the interferometer. The sensitivity is thereby proportional to the path length difference and can be adjusted accordingly. However, optical constraints limit the achievable sensitivity and possibly more than DGV, the application of the technique was so far confined to high speed flows (Seiler et al. 2008). Lu et al. (2007) recently adapted the DPV approach to an actively stabilized Mach-Zehnder setup and demonstrated its capabilities on a rotating disc and a free jet.

Near-resonant interferometry (Landolt and Roesgen 2006) exploits the dispersive properties of an ALF to determine the Doppler frequency shift. In one path of an imaging interferometer, a vapor filter cell is included. With the illumination frequency tuned to a region of anomalous dispersion in the vicinity of a resonant transition, the optical path length difference between the two legs of the interferometer becomes strongly dependent on frequency. Any frequency shift will thus result in a phase distortion of the wave front. The information in form of local deformations in a carrier-fringe pattern is recorded at the image plane of the interferometer.

Compared to DPV, the sensitivity can be significantly enhanced (Landolt and Roesgen 2006). The approach results inherently in a single-camera system in which the velocity recordings are largely independent of the amplitude behavior or linearity of the imaging sensor. This makes the method capable of capturing DGVvelocity ranges and of using ICCD cameras to record weak signals.

\section{Investigation of near-resonant interferometry}

Resonant absorption and dispersion form real and imaginary part of a linear and causal physical process (i.e., the complex refractive index). A modified Hilbert transform pair, the Kramers-Kronig relations, can be formulated that 
relates the two optical properties (Peiponen et al. 1998). The dispersion scales with the absorption coefficient at line center and therewith depends on vapor pressure and temperature. If a Gaussian line shape is assumed, the change in refractive index scales with the steepness of the frequency cutoff. As a result, the dispersion in different materials can be compared based on the figure of merit introduced by Miles et al. (2001) for DGV absorption cells. Of the materials listed, iodine vapor promises the highest gradients in the refractive index.

With its wide use in optical metrology (e.g., DGV), the absorption properties of iodine vapor are well documented. Together with the Kramers-Kronig relations, this allows an accurate prediction of the dispersive properties. In the present study, the absorption model presented by Forkey et al. (1997) is used to calculate the transmission of $I_{2}$ at Nd:YAG laser wavelengths. The model assumes a Gaussian line shape, thereby neglecting pressure broadening effects. The non-resonant background absorption which is not included in Forkey's model is accounted for in the present work using the constants tabulated by Tellinghuisen (1982).

Figure 2 shows the calculated transmissivity and the refractive index for the operating conditions of the used iodine vapor cell (cf. the description of the cell in Sect. 3 for more details). From the plot, the strongest absorption line is identified as no. 1111 at a wavenumber of approximately $18,788.45 \mathrm{~cm}^{-1}$. The maximum gradient of refractive index is found on the blue side (high-frequency positive slope) of this line. For maximum sensitivity, the
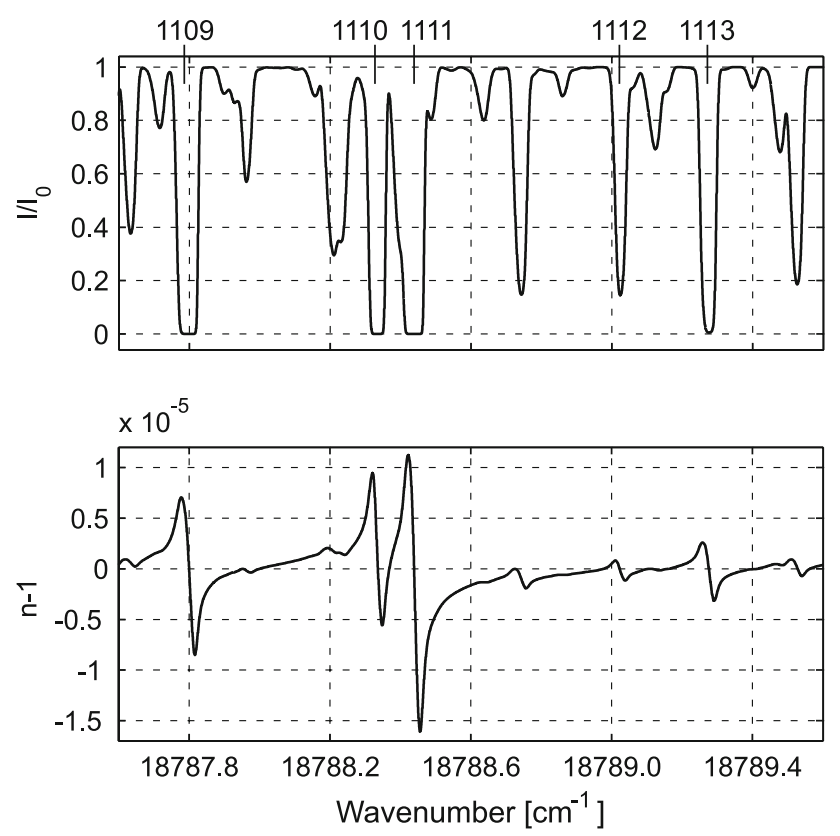

Fig. 2 Predicted transmissivity and refractive index of the Iodine cell used. Line numbering according to Gerstenkorn and Luc (1978) laser operating wavelength was set onto this slope for the subsequent measurements.

When light passes through the dispersive element, frequency changes are transformed into changes in the phase distribution of the wavefront. The present setup uses the iodine cell in one leg of an imaging Michelson interferometer (Fig. 3) to record the phase. At the image plane of the interferometer, the phase difference $\Delta \phi$ of two light waves is a function of the optical path difference, the angular frequency of the light $\omega$ and the speed of light $c$. With the dispersive cell in place, the optical path length difference becomes a function of frequency. The sensitivity of the setup can be defined as the phase shift of the interference pattern due to a change in frequency:

$\frac{\partial \Delta \phi}{\partial \omega}=\frac{2 l}{c}\left[\omega \frac{\partial(n(\omega)-1)}{\partial \omega}+(n(\omega)-1)\right]+\frac{2 L}{c}$,

where $l$ is the length of the dispersive element, $n(\omega)$ its frequency-dependent refractive index and $L$ the geometrical path difference. Applying Eq. 2 to the refractive index data, the characteristics of the setup can be studied (Landolt and Roesgen 2006). As in DGV, the choice of the absorption line, the filter cell and its operating conditions allow for some adjustment. In addition to that, introducing a non-zero geometrical path length difference would allow to combine the present technique with DPV and thus to increase both sensitivity and velocity dynamic range of the system.

Comparison of the theoretical data with the measured values confirms the prediction (Fig. 4). The calibration was conducted on a rotating disc with the laser sweeping through the frequency band of interest. The actual frequency value was determined during the sweep using the output of the frequency monitoring system of the laser (cf. the description of the laser system in the Sect. 3). The magnitude of the system sensitivity is somewhat overestimated by theory which is in all likelihood due to neglecting

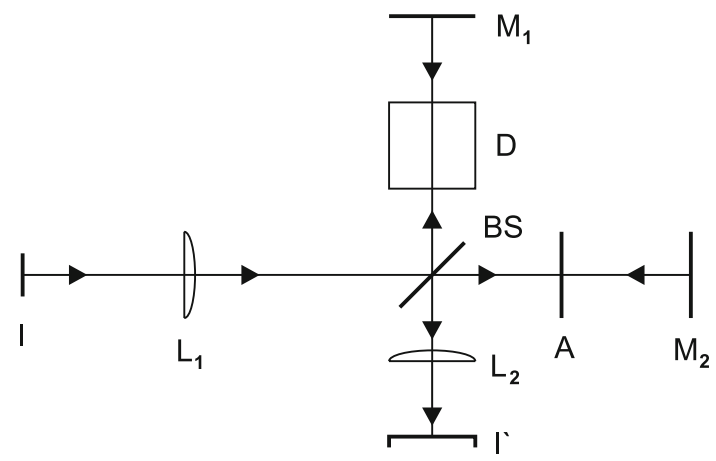

Fig. 3 Michelson interferometer with a dispersive element D. $M_{1}$ and $\mathrm{M}_{2}$ are mirrors, BS the beam splitter and A an attenuator. The lenses $\mathrm{L}_{1}$ and $\mathrm{L}_{2}$ form an image of the object plane $\mathrm{I}$ on both mirrors and in the image plane $\mathrm{I}^{\prime}$ 


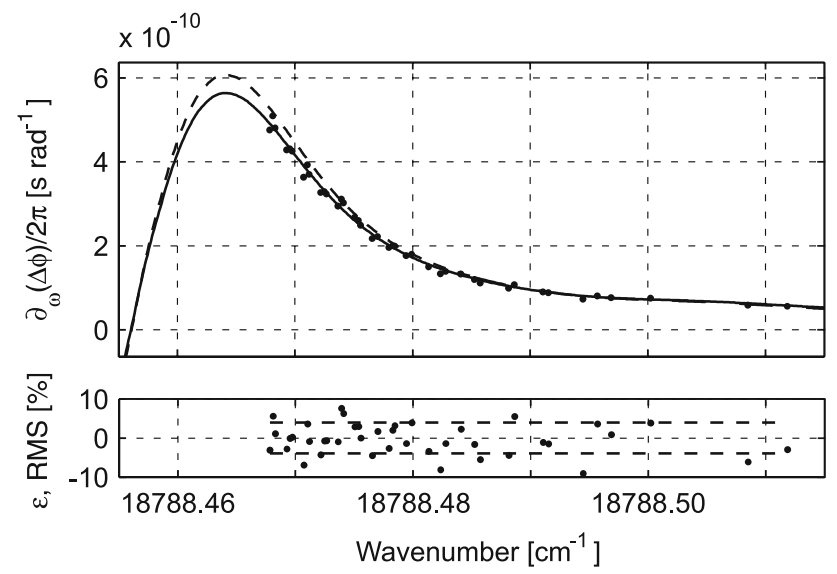

Fig. 4 The measured sensitivity (filled circle) compared to the theoretical prediction (broken line) according to Eq. 2 and the curve fitted to the experimental data (unbroken line) (top) and the relative deviation of each measurement point from the fitted curve (filled circle) and their combined RMS value (dashed line) (bottom)

pressure broadening in the absorption model. To obtain the relation of phase shift angle versus frequency shift from the calibration, the theoretical curve was least-squares fitted to the experimental data by a scaling factor of 0.918 and an offset of $7.04 \times 10^{-12} \mathrm{~s} \mathrm{rad}^{-1}$.

\section{Methods}

The experimental setup shown in Fig. 5 follows closely the layout of Fig. 3. An F-mount adaptor connects the interchangeable entry zoom lens to a $75 / 200 \mathrm{~mm}$ AR-coated spherical lens pair. This pair relays the intermediate image via a non-polarizing beam splitter cube onto the surface of the two mirrors. Two kinematic mounts allow for adjustment of the fringes' orientation and spacing by tilting and

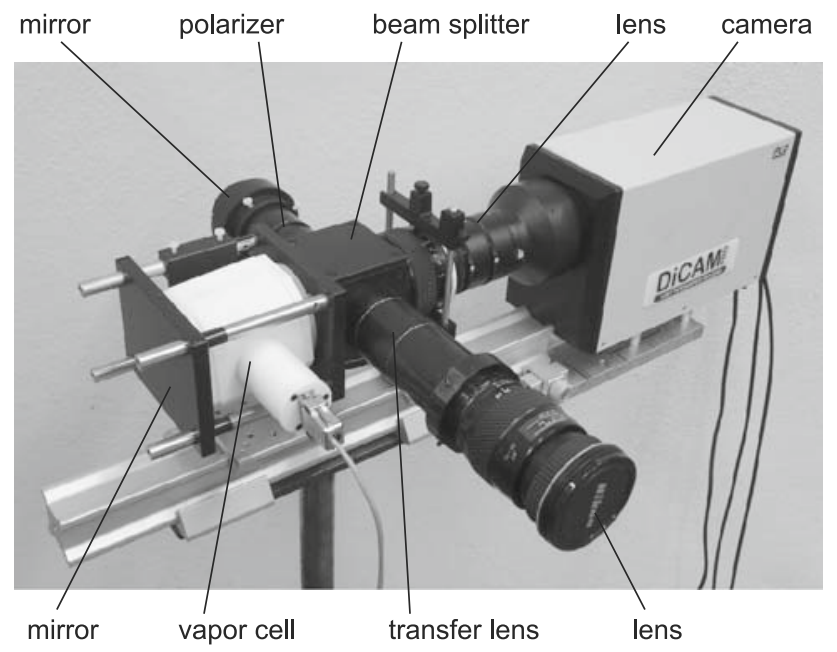

Fig. 5 Experimental setup with the intensified camera rotating the beam splitter and the end mirror in the reference leg, respectively. To increase the fringes' depth of modulation, the intensity of the two beams can be balanced by the variable attenuation of two rotating polarizers in the reference leg. The interference pattern of the recombined images is formed by imaging the two mirror surfaces onto the camera sensor with a standard $85 \mathrm{~mm} \mathrm{f/1.4} \mathrm{lens.}$ Depending on the sensor size of the camera, extension tubes of different lengths are used as specified below. The geometrical path length of each leg can be adjusted independently in order to obtain the desired light focussing. To prevent internal reflections, the Iodine cell is tilted by approx. $5^{\circ}$.

The constant-density iodine vapor cell consists of a 4-cm diameter glass tube with a length of $5 \mathrm{~cm}$, sealed off at both ends with optically flat, AR-coated glass windows. A stem protruding on one side acts as cold finger. The complete glass body is placed in an electrically heated copper structure. For the present study, a cell filled at $60 \mathrm{C}^{\circ}$ (equivalent to a vapor pressure of 4.26 Torr) was operated at stem temperatures of $74 \mathrm{C}^{\circ}$. The cell and the associated temperature stabilization were purchased from the Institute of Propulsion Technology of the German Aerospace Research Establishment (DLR) in Cologne.

For the free jet measurements, a PULNIX TM-1010 monochrome 10-bit camera with a 1,008 $\times 1,008$ pixel $1^{\prime \prime}$ $(9.1 \times 9.2 \mathrm{~mm}) \mathrm{CCD}$ sensor was connected to the interferometer by a $27.5-\mathrm{mm}$ extension tube. Due to the low signal levels and in order to demonstrate the performance of an intensified camera in the setup, a PCO DiCAM-PRO ICCD camera was used for the wind tunnel campaign. Its third generation intensifier has a GaAsP photocathode with a quantum efficiency of $29.5 \%$ counts per photoelectron and an effective photocathode area of $13.5 \times 10 \mathrm{~mm}$. The output phosphor type is P46. The $2 / 3^{\prime \prime}(8.6 \times 6.9 \mathrm{~mm})$ CCD sensor has a resolution of $1,280 \times 1,024$ pixels and 12-bit dynamic range. The true dynamic range was lower due to the increased noise floor in a low-illumination/highfrequency configuration. This reduction, however, is not critical because the velocity information is contained in a spatial pattern and not primarily in the intensity information. The camera was connected to the interferometer using a 55-mm extension tube. Replacing the camera required no re-calibration of the system whatsoever.

The phase information is recorded in the form of carrierfringe images by the imaging sensor. A reference image, taken at wind-off conditions, allows to remove the carrier phase by subtraction. At the same time, the effects of optical imperfections are cancelled. The phase distribution of measurement and reference images is reconstructed by convolving each image with a 2D Gabor filter pair (Jähne 2005) and computing the inverse tangent from the filter responses. The filter pair is defined by a harmonic function 
modulating a Gaussian and acts as a sharp band-pass filter. The peak filter frequencies are determined by locating the maxima of the carrier-fringe frequency in frequency space. The subsequent unwrapping of the phase is achieved by solving the Poisson equation in a least-squares sense (Ghiglia and Romero 1994). Possible errors due to phase jumps in the fringe image close to $\pi$ (e.g., on the disc rim), can be avoided effectively by introducing a separating zero-valued weighting line in the unwrapping process as suggested by the same authors. Finally, the reference image phase distribution is subtracted from the measurement phase. Figure 6 illustrates the process. With the relation between phase and frequency shift obtained from the calibration runs, the Doppler shift distribution is calculated and the measured component of the velocity field can be computed according to Eq. 1. The time required by the non-optimized Matlab-code to process a single fringe image was approx. $30 \mathrm{~s}$ on a current workstation wherein the phase-unwrapping required most of the effort.

The flow was illuminated using a light-sheet generated with a tunable frequency-doubled, injection-seeded longpulse Nd:YAG laser running at $10 \mathrm{~Hz}$. The laser is a customized Surelite PIV laser system from Continuum with increased $4.05-\mathrm{m}$ cavity length to reduce the Fourier limit of the bandwidth. The output energy of the system is $100 \mathrm{~mJ}$ at $532 \mathrm{~nm}$, the pulsewidth $40 \mathrm{~ns}$ and the linewidth (according to the manufacturer) better than $30 \mathrm{MHz}$. The thermal tuning range is $35 \mathrm{GHz}$ and the frequency drift approx. $200 \mathrm{MHz} /{ }^{\circ} \mathrm{C}$. Frequency overlap between seeder and host laser is achieved by dithering the host cavity length to minimize the build-up time which results in a pulse-to-pulse variation of $2.2 \mathrm{MHz}$.

Due to the poor temporal stability of Nd:YAG systems (Meyers et al. 2001), the output frequency has to be monitored: A holographic beam sampler guides $1 \%$ of the laser output onto 2 fast photodiodes. One of the diodes is placed behind a reference iodine cell, the other acts as an intensity reference. For each measurement image, the signal of the two photodiodes is recorded which allows to determine the instantaneous output frequency and to verify the correct seeding of the laser. For sufficient fringe contrast at low seeding levels, the laser was set to a cell transmission of approx. 0.4 for the measurements.
The free jet was formed by pressurized air expanding through a convergent circular nozzle with $4^{\circ}$ half angle and a diameter $d=0.017 \mathrm{~m}$ into an acrylic glass cube with an edge length of $47 d$. Driven by a closed-loop blower, the jet reached an exit speed of $110 \mathrm{~ms}^{-1}$. Seeding was achieved with a commercially available fog generator vaporizing glycol-based fluid to produce particles with a mean diameter of approximately 1-2 $\mu \mathrm{m}$. Manufacturer's numbers vary but based on the short observed lifetime of the particles, it seems reasonable to pick numbers at the upper end of the size distribution. The entire volume of the cube was seeded prior to the measurement which allowed recording the necessary zero-velocity reference. With the blower running, a significant portion of the particles was trapped during one round-trip. This reduced the seeding density of the jet core to a level significantly below ambience. A branching in the blower suction line connected to the fog generator allowed to add seeding particles to the flow and with this to adjust the particle density ratio between jet core and ambient air in the cube. The adjustment was done visually in order to reach a compromise between signal amplitude and the negative effects of multiple scattering between particles and background luminosity present in densely seeded flows (Willert et al. 2007).

The jet axial velocity profile was surveyed using a 3-mm Pitot-static tube prior to the Doppler measurements. The dynamic pressure was recorded along a vertical cut through the jet core at a distance of $10 d$ from the nozzle and in the laser light-sheet plane without seeding. Verification showed no influence of the seeding on the Pitot measurements. The position was metered with the help of a vernier scale on the linear guide of the Pitot. Temperature effects were corrected with a K-type thermocouple at $6.5 d$ from the Pitot-probe. Both dynamic pressure and temperature were recorded at a rate of $1 \mathrm{kHz}$ and averaged over $20 \mathrm{~s}$.

The observation and illumination angles were chosen for increased light intensity in forward scattering at $59^{\circ}$ and an acceptable Doppler shift of $1.74 \mathrm{MHz} / \mathrm{ms}^{-1}$ (Fig. 7). To allow comparison with the Pitot control measurements, the respective probe tip was traced through a sequence of images recorded at ambient light. During the Doppler measurements, the probe was removed. The interferometer was adjusted to a $0.18 \times 0.18-\mathrm{m}$ field of view and a

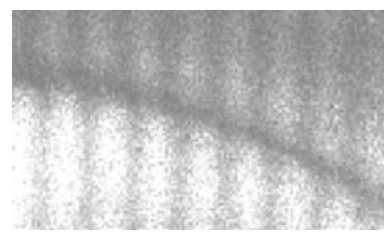

(a)

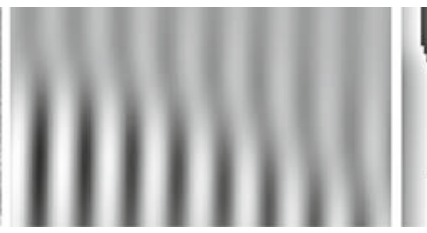

(b)

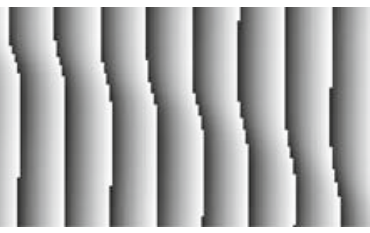

(c)

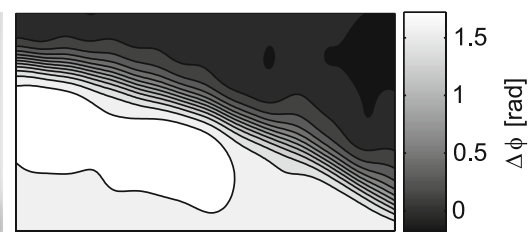

(d)

Fig. 6 The post-processing illustrated. The raw measurement image (a) (showing a detail of the rotating disc), the filtered image (b) (real part), the wrapped phase (c) and the unwrapped phase shift angle (carrier phase removed) 
Fig. 7 Geometrical arrangement for the subsonic jet experiments

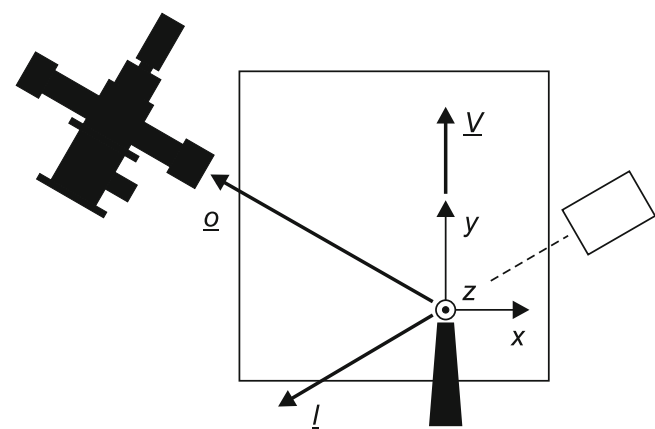

Unit vector at origin $(x, y, z)$ :

$\begin{array}{llll}0 & -0.831 & 0.457 & 0.317\end{array}$

$\begin{array}{llll}l & -0.883 & -0.470 & 0.003\end{array}$

$\begin{array}{cccc}V & 0 & 1 & 0\end{array}$

Scattering Angle: $59^{\circ}$ carrier-fringe wavelength of 8 pixels. The images were processed with a Gabor filter of 12 pixels FWHM for the measurement and 18 pixels for the reference images, respectively. With the reference phase assumed to be approximately smooth, the spatial resolution is governed by the filter applied to the measurement image and was $2 \mathrm{~mm}$ (11 pixels) for the jet experiments (cf. Sec. 5 about the determination of the spatial resolution).

The second set of measurements considered the tip vortex flow of a blunt-edged NACA 0012 wing model with a chord $c$ of $0.2 \mathrm{~m}$ and a half-span of $1 \mathrm{~m}$. The wing was mounted in the large low speed wind tunnel of the Institute of Fluid Dynamics at ETH shown in Fig. 8. The freestream velocity $V_{\infty}$ in the tunnel was set to $45 \mathrm{~ms}^{-1}$ resulting in a measurement Reynolds number of $5.4 \times 10^{5}$ based on the chord of the wing. The laser is guided to the test section by a set of mirrors and a light-sheet optic mounted on a traversing unit. The light sheet was aligned parallel to the tunnel center line and positioned laterally to cut through the vortex core with the wing at $+12^{\circ}$ angle of attack (AOA).

The flow was seeded with vaporized glycol-based fluid added directly downstream of the test section. To limit the negative effects of a large densely seeded measurement volume (i.e., reduced illumination intensity and image contrast and increased background luminosity), the outlet of the smoke generator was positioned such that mainly the area of interest was seeded during the measurements. Global seeding could be achieved by continuously seeding over several round-trips. To record the reference images, the volume of the test section was globally seeded with the tunnel turned off.

The measurement origin was set to the trailing edge of the airfoil (Fig. 8). The sensor covering an area of $2 \times 1.5 c$ was placed at $(c, 1.71 \mathrm{~m}, 0)$ and the spreading lens of the light-sheet optics at $(c, 0,1.35 \mathrm{~m})$. At $x=c$ the direction of observation $o$ was thus perpendicular to the free-stream velocity $V_{\infty}$ and normal to the propagating direction of light. Accordingly, for $x=c$ the measured velocity component was the bisection between $y$ and $z$ axis. In the $x z$ plane of the vortex core (i.e., the light-sheet position), the flow was assumed to have a vanishing vertical $(V z)$ component. The Doppler shift for the circumferential velocity component $V y$ could thus be calculated to $1.88 \mathrm{MHz} / \mathrm{ms}^{-1}$. The carrier fringes were adjusted to a wavelength of 9 pixels, while a filter of 18 pixels FWHM was used on the images for post-processing. The resulting spatial resolution of 17 pixels or $5 \mathrm{~mm}$ is slightly lower than for the jet experiments. The reason for this seems to be the effect of the intensifier's micro-channel plate on the resolution of the camera. The sensor field of view was mapped to the wind tunnel coordinates based on pictures of a calibration target clamped to the wing during setup.

The control measurements were conducted with a 75-mW fp50-shift LDV system from Intelligent Laser Applications (ILA). The probe with 400-mm focal length was mounted in the top window of the tunnel on its own $y z$-traversing unit. Because seeding downstream of the wing did not yield a sufficient amount of counts, seeding was performed using a PIVTEC aerosol seeding generator with 12 Laskin nozzles running with DEHS fluid. This device permitted to seed above ambient pressure in the nozzle of the wind tunnel, $3 \mathrm{~m}$ upstream of the model. The density of the seeding with a mean particle diameter of $0.95 \mu \mathrm{m}$ was considerably lower than for the Doppler measurements. The circumferential component $V y$ of the flow was recorded along the vertical axis through the vortex core at $x / c=1.0$ every $10 \mathrm{~mm}$ and with approximately $120-\mathrm{s}$ sampling time at each point. Due to the particles becoming very scarce near the vortex core, no data could be collected in this region.

\section{Results}

Figures 9 and 10 illustrate the practicability of the technique for flow measurements by the reasonable agreement of the experimental data with the control measurements. The data was averaged over 8 images for the jet and 15 images for the wind tunnel measurements, respectively.

The contour plot of the measured Doppler shift for the free jet in Fig. 9 shows the expected circular distribution in 
Fig. 8 Sketch of the wind tunnel facility including main dimensions in $\mathrm{mm}$ and arrangement of the measurement equipment and the geometrical setup
Fig. 9 Results from the jet flow measurements. The measured averaged frequency shift distribution is shown on the left. The figure on the right compares the averaged measured flow velocity component (unbroken line) with the respective control data (filled circle) including error $\varepsilon$ and RMS values between the individual images
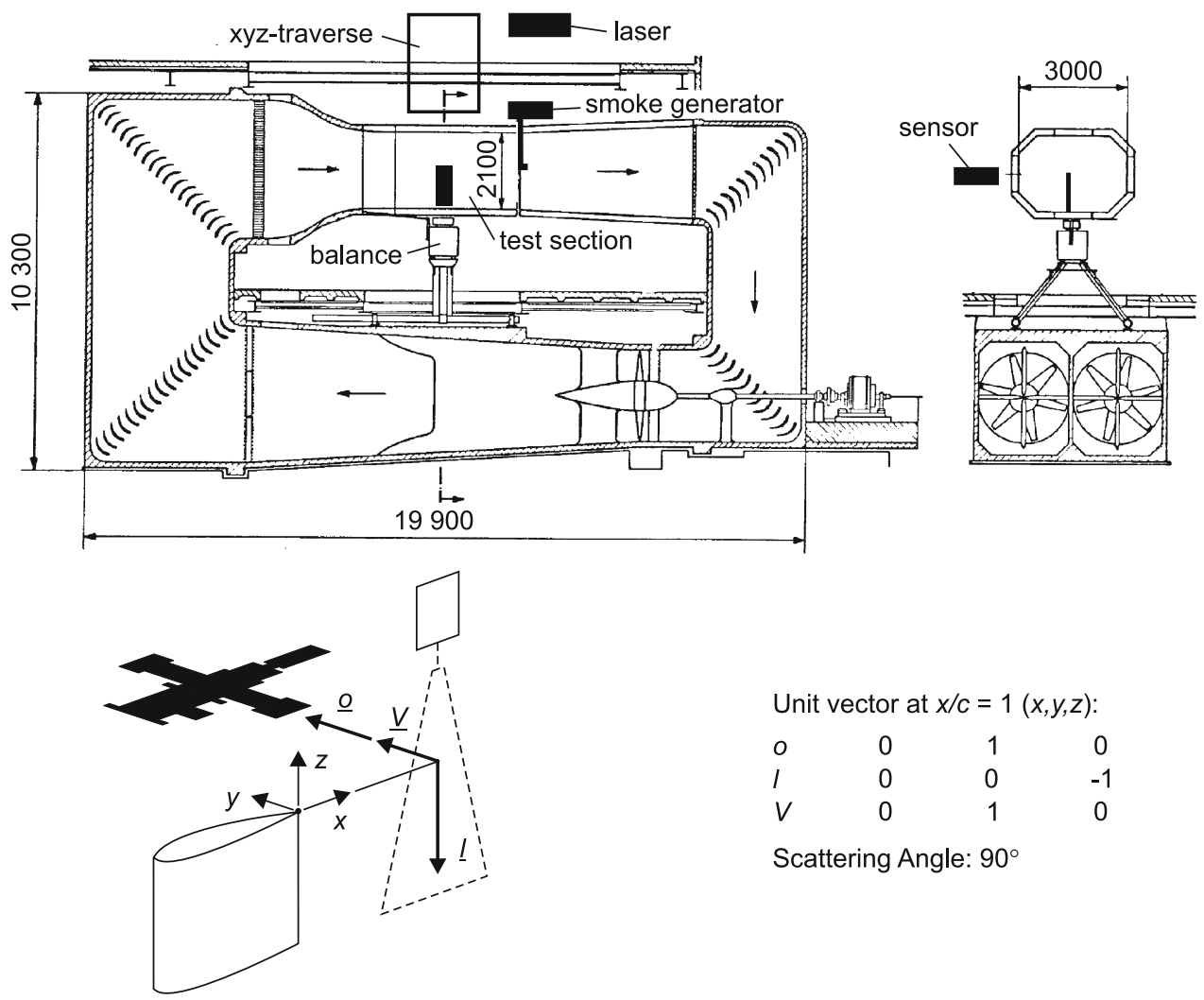

Unit vector at $x / c=1(x, y, z)$ :

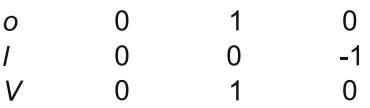

Scattering Angle: $90^{\circ}$
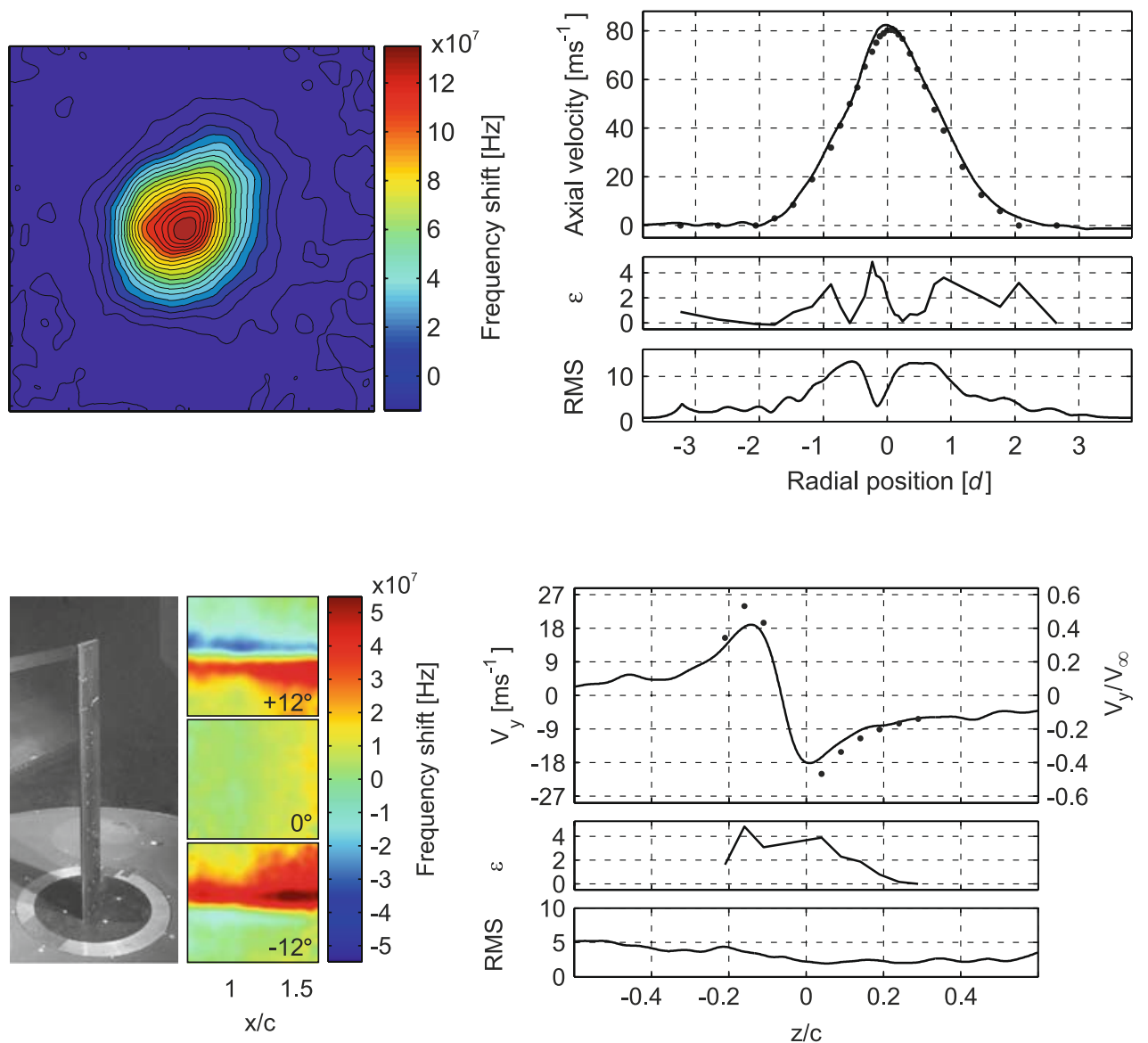

Fig. 10 Results from the tip vortex flow measurements. The measured averaged frequency shift distribution is given on the left for three different angles of attack (flow is from left to right, top $+12^{\circ}$, center $0^{\circ}$, bottom $\left.-12^{\circ}\right)$. The figure on the right compares the measured flow velocity component (unbroken line) with the respective control data (filled circle) including error $\varepsilon$ and RMS values between the individual images 
front of an unshifted background. Along a vertical cut through the jet core, the velocity agrees well with the Pitotstatic tube measurements. The flow velocity is slightly overestimated by the Doppler data, whereas the error appears to scale with the local flow velocity reaching a maximum of $5 \mathrm{~ms}^{-1}$ near the core. With the comparatively short temporal averaging of the Doppler data $(0.8 \mathrm{~s})$, this can originate both from momentary variations in the flow velocity (fluctuations of the same magnitude and time scale could be observed in the time series of the control measurements) or from measurement error. In conventional DGV and as confirmed by the investigations made on the artificial test images, the method-specific measurement error (i.e., due to drift and optical distortions in the interferometer, image quality and post-processing) is largely independent of the velocity magnitude. Furthermore, because of the spatial averaging effect of multiple scattering and the presence of background luminosity, one would expect the Doppler data to be lower than the actual flow velocity (Willert et al. 2007). The observed discrepancy indicates therefore an error in the determination of the laser frequency (e.g., due to a spatially non-uniform frequency distribution), causing the assumed system sensitivity to be too low. The root mean square (RMS) values computed for the series of 8 images is clearly related to the measured velocity distribution. This is a consequence of an increased turbulence level and small-scale fluctuations in image intensity (i.e., seeding density) near the jet core which affect the phase reconstruction.

For the wind tunnel experiments Fig. 10 shows the averaged measured frequency shift for different angles of attack with the field of view cropped to an edge length of $c$. As the sensitivity of the setup for the $x$-component of the flow only vanishes at $x / c=1$, a superimposed slightly negative gradient from left to right would be expected for all angles of attack. Instead, for $0^{\circ}$ angle of attack, a comparably strong positive gradient is observed. This is believed to be a result of the seeding conditions being different for the wind-off images (i.e., more particles in the test section). With more seeding the particle-particle scattering will increase and the background will be illuminated more strongly, both of which will have an averaging effect on the illumination frequency. The observed velocity gradient results from subtracting this more averaged frequency distribution from the measurement image. The increased streamwise variation for $-12^{\circ} \mathrm{AOA}$ can be attributed to a non-symmetric angle between the vortex core and the light sheet for positive and negative angles of attack. If the light sheet is not cutting exactly through the vortex core, the $z$-component of the flow contributes to the Doppler shift.

With the insensitivity to the streamwise $(x)$ component of the flow and vanishing vertical component in the $x z$ plane, the Doppler measurement can be compared to the LDV data at $x=c$. The plot shows that the circumferential component is clearly underestimated by the Doppler data. This smoothing of the velocity gradient is caused both by the finite spatial resolution and by multiple scattering and background effects which spatially average the observed frequency distribution and bias the weakly seeded core regions towards zero frequency shift. Consistent with the observed smoothing of the gradient, the measurement error is $4 \mathrm{~ms}^{-1}$ near the vortex core and appears to decrease away from it. The RMS between the individually processed images decreases slightly with increasing $z$. As this was observed for all angles of attack, it could be due to the light-sheet intensity diminishing along its path through the measurement volume or an increased turbulence level behind the wing.

\section{Discussion}

As no spatial averaging occurs during unwrapping, both the spatial resolution of the technique and the post-processing error are determined in the filtering step. The resolution limit for a carrier-fringe technique is tied to the spatial fringe wavelength and thus to the system optical resolution. To quantify the spatial resolution, the distance required by the technique to rise from 10 to $90 \%$ of a phase step (edge response) is used in the present work. For Gabor filters, an edge response slightly better than the filter size $(0.93$ times the FWHM) was found by processing a $\pi / 2$ phase step with different filter sizes. The minimum FWHM relative to the fringe wavelength was determined by processing test images of different carrier wavelengths with varying filter sizes to be 1.2 times the fringe wavelength. Below this value, the post-processing error started to increase gradually. With the size fixed at 1.2 times the fringe wavelength, the error increased rapidly if fringe wavelengths were lower than 6 pixels in the test images. Based on the specified step response, a maximum spatial resolution of the technique of 7 pixels can thus be expected. The orientation of the fringes relative to the sensor's pixel had no observable effect. The test images were generated by calculating the local carrier phase shift distribution and the absorption of a given velocity field. A combination of Gaussian white noise and multiplicative speckle noise was superimposed.

With decreasing fringe contrast, the uncertainty in the computed phase was observed to increase at first slightly and then to jump up below a critical contrast level. The total failure in phase reconstruction is marked by a random phase output. Its occurrence coincides nicely with the vanishing of the carrier frequency peak in the power spectra of the respective image section. In general, the 
phase reconstruction was found to perform better than with $2-\mathrm{MHz}$ uncertainty or $0.03 \mathrm{rad}$ even with the minimum filter size and at low fringe contrast. Isolated regions with low contrast (e.g., due to non-uniform seeding particle distribution in a turbulent flow) require a larger filter FWHM and thus decrease the spatial resolution.

Intensity variations in the images can bias the phase reconstruction depending on their amplitude and orientation relative to the fringes. The spatial frequency with which the intensity varies has thereby no influence as long as it is distinguishable from the carrier-fringe frequency in Fourier space. No error occurs if the carrier fringes are aligned parallel to the intensity gradients. The effect of the static intensity distribution observed in the flow measurement images ( $\sim 20 \%$ of maximum intensity caused, e.g., by inhomogeneous seeding, the frequency-dependent absorption in the filter cell and optical vignetting) is negligible compared to other error sources. Nevertheless, for the tip vortex measurement, the fringes were oriented parallel to the flow-induced intensity gradients. Transient, small-scale intensity variations (e.g., due to flow turbulence) will somewhat increase the root mean square (RMS) values of the measurements. Velocity gradients have only an indirect influence on the phase reconstruction. Due to the frequency-dependent absorption of the filter cell, the corresponding Doppler shifts cause a variation in intensity which can affect the phase computation as discussed above.

For the processing of the actual jet and tip vortex measurement images, the filter size had to be found iteratively according to the image quality. The size was thereby gradually reduced until the computed phase started to develop a wavy pattern with a spatial wavelength close to the filter FWHM. During this process, an increase in the velocity gradients consistent with the decrease in spatial averaging was observed.

During the setting up of the experiments, the common global Doppler problem of finding a compromise between seeding intensity, scattering angle and Doppler sensitivity proved time consuming. Installing and adjusting the interferometer was straightforward. Switching cameras involved only re-focussing and no camera-specific calibration of the system was necessary. When decreasing the carrier-fringe wavenumber to achieve high spatial resolution, it became increasingly difficult to maintain high fringe contrast throughout the image. The tilting of one mirror inclines the image plane at the camera position which moves part of the image out of focus. This was observed to let the measurement accuracy deteriorate quickly in the respective regions. Increasing the $f$-number was an option only for experiments with high light levels.

The observed uncertainty in the calibrated sensitivity was $4 \%$ (Fig. 4) which is equivalent to $8.2 \mathrm{MHz}$. This reflects primarily the uncertainty in laser frequency determination, the disc speed readout error $(<1 \%)$ and the post-processing error which all are, besides the disc readout error, of an absolute nature. This seems thus to represent reasonably well the performance of the present setup. For the two flow measurements performed, $8 \mathrm{MHz}$ would correspond to an uncertainty of $\sim 5 \mathrm{~ms}^{-1}$, which is confirmed by the experimental data presented. The measurements on the free jet show that velocities down to $2 \mathrm{~ms}^{-1}$ can be resolved.

With only a few seconds delay between the acquisition of reference and measurement images, the drift of the interferometer could be neglected. Non-negligible drift would result in a constant phase offset between reference and measurement images. Its correction would require knowledge of the velocity in a single reference point or to actively stabilize the interferometer as done by $\mathrm{Lu}$ et al. (2007).

As reported by Willert et al. (2007), multiple scattering between particles has a spatial averaging effect by reducing image contrast. The same authors suspect background luminosity (caused by light scattered omnidirectionally by the particles in the light sheet) to lead to an underestimation of the measured values. In the present work, for both jet and tip vortex experiments the observed Doppler shifts decreased with increasing seeding levels. Setting the seeding to a minimum had therefore to be done carefully in an iterative way. The aim of the jet experiments was to measure both jet core and the surrounding near-stationary background which required the entire measurement volume to be seeded. To limit the effect of background luminosity, the seeding level in the jet had to be equal or higher than in the ambient volume. Without additionally seeding the core, an underestimation of the velocity up to $30 \%$ could be observed. A similar effect was apparent in the tip vortex experiment where the flow near the vortex core was only scarcely seeded compared to the rest of the measurement volume. Unlike for the jet, increasing the seeding level in the core was not feasible. Additionally, the restricted optical access to the wind tunnel allowed only working with less efficient scattering angles around $90^{\circ}$ which increased the relative importance of the background signal. With the rather coarse LDV measurement grid it is possible that the discrepancy between true flow velocity and Doppler data is more important than stated. Values in literature vary, but numbers just above 0.6 for $V y / V_{\infty}$ can be found which could imply an error of $9 \mathrm{~ms}^{-1}$ instead of the $4 \mathrm{~ms}^{-1}$ stated above. Given the strong velocity gradients of the flow, it is believed that the observed underestimation illustrates the combined impact of particle-particle scattering, background luminosity and relatively coarse spatial resolution on the measurements.

Consistent with the findings of other authors (Meyers et al. 2001) an important source of error was identified in 
the spatial frequency variations in the laser beam. Changes in the determined sensitivity up to $15 \%$ were sometimes observed when recoded over a period of days or weeks on the rotating disc. This is believed to be caused by a changing spatial frequency distribution in the laser beam. According to the effective distribution, the local illumination frequency and therefore sensitivity was apparently higher or lower than estimated by the monitoring system. The variations observed for the jet and tunnel experiments were smaller which can be explained by the averaging nature of the light-sheet optics. The correction of an error in assumed sensitivity would require additional information about the flow, e.g., a reference point of high known velocity.

\section{Conclusions}

The feasibility to measure global Doppler frequency shifts using the anomalous dispersion of an atomic line filter (ALF) in an interferometric setup could be demonstrated. An experimental calibration of the system allowed to assess the prediction of the iodine cell's dispersion and to determine the system sensitivity. The observed discrepancy between prediction and calibration could be attributed to neglecting pressure broadening in the absorption model. Velocity measurements on a free jet and a tip vortex flow in a medium-sized wind tunnel were conducted. For the measurement in the wind tunnel, an intensified camera was used. Both experiments were found to be quite challenging in terms of seeding conditions, velocity gradients, facility size and the use of a pulsed light source. Consequently, the obtained results represent the true performance of the method in a non-laboratory environment.

The technique was found capable of reproducing the flow velocity distribution with reasonable accuracy. Based on the calibration data, the uncertainty could be estimated to $8 \mathrm{MHz}$ or $5 \mathrm{~ms}^{-1}$. Aside from the secondary effect of reduced fringe contrast due to frequency shifts towards the absorption line center, this value is independent of velocity. Two important error sources that are not specific to the method but common to Doppler global techniques are thereby not fully accounted for: the determination of the illumination frequency and the effects of multiple scattering between particles and background luminosity.

The phase reconstruction could be identified as the main error source in the data analysis. By processing synthetic test images, a spatial resolution of 7 pixels with an uncertainty of less than $2 \mathrm{MHz}$ or $0.03 \mathrm{rad}$ was achieved. Images with a high noise level, low fringe contrast and/or important intensity variations require a larger filter sizes which decreases the spatial resolution. The resolution achieved for jet and tip vortex measurements was 11 pixels or $2 \mathrm{~mm}$ and 17 pixels or $5 \mathrm{~mm}$, respectively. The comparatively low resolution of the tip vortex data contributes to some degree to the observed underestimation by smoothing the velocity profile.

Compared to DGV, the technique has its assets and drawbacks. Given the described equivalence between absorption and dispersion, the available information content is not expected to have increased. Consistent with this, the accuracy of the post-processing suggests that with a better light source (e.g., an argon ion laser, a cw-laser often used for DGV), the performance would be comparable to that of a conventional DGV setup ( $\sim 2 \mathrm{MHz}$ accuracy). The main advantage of near-resonant interferometry is clearly the single-camera capability. Image alignment and camera calibration errors which can be the source of important errors in DGV are negligible. Furthermore, the setup is less sensitive to the characteristics of the imaging sensor. This allowed to use an intensified camera at low light scattering levels without re-calibrating the system. Negative aspects are the opto-mechanical complexity of an interferometer with its liability to drift and vibration and the expensive post-processing with a spatial resolution limited to 7 pixels. Phase jumps close to $\pi$ (e.g., at the disc rim) may require increased pre-processing effort. An additional advantage over DGV remains to be experimentally verified: by introducing a geometrical path length difference in the interferometer it should be possible to tune the system characteristics and to increase the velocity dynamic range considerably compared to DGV which is restricted to the shoulder of an absorption line.

With DPV the method shares the complexity of an interferometric setup, the processing of carrier-fringe images and the large measurement range but the sensitivity is significantly enhanced. The disadvantages are the light absorption at frequencies close to a line center, the increased calibration effort and the requirements on the laser stability and bandwidth.

In a next step, the method should be assessed using a stabilized cw-laser which would allow to investigate the contributions of the different error sources more closely. The further development of the setup should minimize the influence of the environmental conditions (e.g., temperature-induced drift of the interferometer) and reduce the internal optical losses. As the fringe contrast depends on correct focusing, a set of aspherical lenses should be fitted. It is likely that the accuracy of the phase reconstruction can be improved by further development of the described algorithm. Sub-pixel interpolation should be applied to locate the maxima in frequency space in order to determine the frequencies of the Gabor filter more precisely. The bias of the image intensity distribution on the phase reconstruction and background corrections should be assessed in detail. Other phase-sensing principles like phase-stepping 
interferometry are conceivable, but would come at the cost of lower temporal resolution and increased hardware complexity.

With its single-camera capacity, large dynamic range and the flexibility in the choice of the dispersive medium and geometrical path length difference, the present technique adds a promising variant to the class of Doppler global techniques.

\section{References}

Fischer A, Büttner L, Czarske J, Eggert M, Grosche G, Müller H (2007) Investigation of time-resolved single-detector Doppler global velocimetry using sinusoidal laser frequency modulation. Meas Sci Technol 18:2529-2545

Ford HD, Nobes DS, Tatam RP (2001) Acousto-optic frequency switching for single-camera planar Doppler velocimetry. Proc Soc Photo Opt Instrum Eng 4448:272-282

Forkey JN, Lempert WR, Miles RB (1997) Corrected and calibrated $\mathrm{I}_{2}$ absorption model at frequency-doubled Nd:YAG laser wavelengths. Appl Opt 36(27):6729-6738

Gerstenkorn S, Luc P (1978) Atlas du spectre d'Absorption de la Molecule d'Iode, 14,800-20,000 $\mathrm{cm}^{-1}$. Laboratoire Aimé-Cotton CNRS II, Orsay

Ghiglia DC, Romero LA (1994) Robust two-dimensional weighted and unweighted phase unwrapping that uses fast transforms and iterative methods. J Opt Soc Am A 11(1):107-117

Jähne B (2005) Digital image processing. 6 th rev. and extended edn. Springer, Heidelberg
Komine H, Brosnan SJ, Litton AB, Stappaerts EA (1991) Real-time Doppler global velocimetry. In: AIAA 29th Aerospace Sciences Meeting, Reno, Nevada, paper 91-0337

Landolt A, Roesgen T (2006) Global Doppler frequency shift detection with near-resonant interferometry. In: 13 th Int Symp. on applications of laser techniques to fluid mechanics, Lisbon, Portugal, p 21.7

Lu Z-H, Charrett TOH, Ford HD, Tatam RP (2007) Mach-Zehnder interferometric filter based planar Doppler velocimetry (MZIPDV). J Opt A Pure Appl Opt 9(11):1002-1013

Meier AH, Roesgen T (2009) Heterodyne Doppler global velocimetry. In: 14th international symposium on applications of laser techniques to fluid mechanics, Lisbon, Portugal, p 7.3.4. Exp Fluids. doi:10.1007/s00348-009-0647-0

Meyers JF (1995) Development of Doppler global velocimetry as a flow diagnostics tool. Meas Sci Technol 6:769-783

Meyers JF, Lee JW, Schwartz RJ (2001) Characterization of measurement error sources in Doppler global velocimetry. Meas Sci Technol 12:357-368

Miles RB, Yalin AP, Tang Z, Zaidi SH, Forkey JN (2001) Flow field imaging through sharp-edged atomic and molecular 'notch' filters. Meas Sci Technol 12:442-451

Peiponen KE, Vartiainen EM, Asakura (1998) Dispersion, complex analysis and optical spectroscopy. Springer,

Seiler F, Oertel H (1983) Visualization of velocity fields with Dopplerpictures. In: 3rd Int. Symp. on flow visualization, Ann Arbor

Seiler F, George A, Srulijes J, Havermann M (2008) Flow Progress in Doppler picture velocimetry (DPV). Exp Fluids 44:389-395

Tellinghuisen J (1982) Transition strengths in the visible-infrared absorption spectrum of $\mathrm{I}_{2}$. J Chem Phys 76(10):4736-4744

Willert C, Stockhausen G, Klinner J, Lempereur C, Barricau P, Loiret P, Raynal JC (2007) Performance and accuracy investigations of two Doppler global velocimetry systems in parallel. Meas Sci Technol 18:2504-2512 